Check for updates

Cite this: Phys. Chem. Chem. Phys., 2018, 20, 18207

Received 14th February 2018 Accepted 28th May 2018

DOI: $10.1039 / c 8 c p 01064 a$

rsc.li/pccp

\section{Average orientation of a fluoroaromatic molecule in lipid bilayers from DFT-informed NMR measurements of ${ }^{1} \mathrm{H}-{ }^{19} \mathrm{~F}$ dipolar couplings $\dagger$}

\begin{abstract}
Eleri Hughes, John M. Griffin, (D) Michael P. Coogan (D) and David A. Middleton (DD*
Fluorine is often incorporated into the aromatic moieties of synthetic bioactive molecules such as pharmaceuticals and disease diagnostics in order to alter their physicochemical properties. Fluorine substitution may increase a molecule's lipophilicity, thereby enabling its diffusion across cell membranes to enhance bioavailability or to exert a direct physiological effect from within the lipid bilayer. Understanding the structure, dynamics and orientation of fluoroaromatic molecules in lipid bilayers can provide useful insight into the effect of fluorine on their mode of action, and their interactions with membrane-embedded targets or efflux proteins. Here we demonstrate that NMR measurements of ${ }^{19} \mathrm{~F}$ chemical shift anisotropy combined with ${ }^{1} \mathrm{H}-{ }^{19} \mathrm{~F}$ dipolar coupling measurements together report on the average orientation of a lipophilic fluoroaromatic molecule, 4-(6-fluorobenzo[d]thiazol-2-yl)aniline (FBTA), rapidly rotating within a lipid bilayer. The ${ }^{19} \mathrm{~F}$ chemical shift tensor orientation in the molecular frame was calculated by density functional theory and corroborated by ${ }^{1} \mathrm{H}-{ }^{19} \mathrm{~F}$ PISEMA NMR. It was then possible to analyse the line shapes of proton-coupled and proton-decoupled ${ }^{19} \mathrm{~F}$ spectra of FBTA in chain perdeuterated dimyristoylphosphatidylcholine (DMPC- $d_{54}$ ) bilayers to restrict the average axis of molecular reorientation of FBTA in the bilayer to a limited range orientations. This approach, which exploits the high sensitivity and gyromagnetic ratios of ${ }^{19} \mathrm{~F}$ and ${ }^{1} \mathrm{H}$, will be useful for comparing the membrane properties of related bioactive fluoroaromatic compounds.
\end{abstract}

\section{Introduction}

Fluorine is becoming increasing prevalent in pharmaceuticals and agrochemicals owing to its ability to influence physicochemical properties such as molecular dipole moments, $\mathrm{p} K_{\mathrm{a}}$ and lipophilicity, which can have profound effects on biological activity. ${ }^{1}$ Lipophilicity is an important property of bioactive molecules, which enables them to cross the blood-brain barrier and to enter, diffuse within, or cross cell membranes to reach their biological target. Drug-lipid interactions may also induce a direct biological response, by destabilising cell membranes to induce endocytosis or to promote fusion with liposomal delivery agents. Understanding the structural and dynamic properties of bioactive molecules within lipid bilayers is fundamentally important for assessing their potential as pharmaceuticals or as diagnostics, for example. Small molecules can diffuse laterally within the plane of a lipid bilayer and rotationally about an axis or axes that define the orientation of the molecule with respect

Department of Chemistry, Lancaster University, Lancaster, LA1 4YB, UK.

E-mail: d.middleton@lancaster.ac.uk; Tel: +44 1524594328

$\dagger$ Electronic supplementary information (ESI) available. CCDC 1584785. For ESI and crystallographic data in CIF or other electronic format see DOI: 10.1039/ с8ср01064a to the bilayer normal. The orientational preference of a molecule within cell membranes can not only influence its activity, but may also affect its interaction with efflux transporters that export drugs from the bilayer back into the blood-stream. ${ }^{2}$ It is estimated that as many of $30 \%$ of new approved drugs contain fluoroaromatic or fluoroalkyl groups. ${ }^{3}$

NMR is particularly well-suited to the study of molecular structure, orientation and dynamics of biomolecules within lipid bilayers represented by large multilamellar or unilamellar vesicles. For small, drug-like organic molecules in large vesicles, where rotational diffusion within the lipid bilayer is rapid and the rates of vesicle tumbling and drug lateral diffusion within the bilayer are slow, the anisotropic nuclear spin interactions (chemical shielding, dipole-dipole and quadrupolar for $I \geq 1$ ) are averaged in a predictable way. ${ }^{2,4-8}$ NMR line shape analysis provides information about the nature of the dynamic process giving rise to the scaled interactions, such as the average orientation of the lipophile relative to the bilayer normal. These measurements often require isotope labelling with ${ }^{13} \mathrm{C},{ }^{15} \mathrm{~N}$ or ${ }^{2} \mathrm{H}$ to enhance sensitivity, which can be costly and technically demanding. ${ }^{19} \mathrm{~F}$ is an attractive nucleus for studies of drug-membrane interactions by virtue of its $100 \%$ isotopic abundance, high receptivity, high gyromagnetic ratio, and low background in endogenous biological materials. ${ }^{6}$ 
Here we report a new approach exploiting NMR measurements of ${ }^{19} \mathrm{~F}$ chemical shift anisotropy and ${ }^{1} \mathrm{H}-{ }^{19} \mathrm{~F}$ dipolar coupling, combined with DFT calculations, to determine the average orientation of lipophilic solutes in lipid bilayers. The combination of ${ }^{19} \mathrm{~F}$ CSA measurements and ${ }^{1} \mathrm{H}-{ }^{19} \mathrm{~F}$ dipolar coupling measurements could, if validated, provide a useful approach for determining molecular orientations of fluorinated drugs in lipid bilayers, by offering high sensitivity (and relatively short measurement times) without requiring isotope enrichment. To test this approach, we use as a model system the lipophilic compound 4-(6-fluorobenzo[d]thiazol-2-yl)aniline (FBTA) (Fig. 2A), which is a fluorinated analogue of the lipophilic Pittsburgh compound $\mathrm{B}$ used for imaging amyloid plaque deposition in the brain and which must cross the blood-brain barrier. ${ }^{9}$

\section{Methods and materials}

\section{Preparation of membrane samples}

For experiments in lipid bilayers, FBTA (Fluorochem Ltd) was recrystallized from $50 \%$ ethanol:water to remove a catalytic impurity. The compound ( $1 \mathrm{mg}$ ) was dissolved in chloroform : methanol $(50: 50)$ with a 20 -fold molar excess of DMPC-d $\mathrm{d}_{54}$ (Avanti Polar Lipids, Inc.) and dried to a thin film in a round bottom flask under nitrogen and then under high vacuum. Multilamellar vesicles were produced by resuspending the film in water, subjecting to 5 freeze-thaw cycles and centrifuging in a bench top centrifuge to remove excess liquid. The pellet was transferred to a $3.2 \mathrm{~mm}$ diameter zirconium rotor and lyophilised overnight. The rotor was fitted with a cap for NMR analysis. The preparation of hydrated membranes followed the same procedure but omitting the final lyophilisation step.

\section{Crystallisation of FBTA}

For crystal structure determination and NMR analysis of the solid, FBTA was recrystallized as a methanol solvate. Commercially sourced FBTA (Fluorochem Ltd) was initially purified by removal of impurities which crystallised first upon cooling of the minimum volume of hot ethanol required to dissolve the sample from hot ethanol followed by precipitation of the FBTA by addition of an equal volume of water. Crystals suitable for X-ray diffraction were then grown by repeatedly crystallising from hot methanol, removing the supernatant liquid and repeating the process 4 times. A suitable crystal was selected and mounted on a Rigaku SuperNova diffractometer with and Atlas detector. The crystal was kept at 100.01(10) K during data collection. The crystallographic data set was solved and refined using the SHELX suite of software. ${ }^{10}$

\section{NMR analysis}

All measurements were performed on a Bruker Avance III spectrometer with a $89 \mathrm{~mm}$ bore magnet operating at $9.3 \mathrm{~T}$. A Bruker quadruple resonance (HFXY) magic-angle spinning probe tuned simultaneously to ${ }^{19} \mathrm{~F}$ and ${ }^{1} \mathrm{H}$ was used for all measurements, without sample spinning except where stated. The recycle delay was $2 \mathrm{~s}$ for measurements on the membrane samples and $4 \mathrm{~s}$ for measurements on solid FBTA.
The proton-decoupled ${ }^{19} \mathrm{~F}$ spectra of solid FBTA and the lyophilised membrane sample were obtained with an initial $2.5 \mathrm{~ms} 90^{\circ}$ pulse at the frequency of ${ }^{1} \mathrm{H}$ followed by $2 \mathrm{~ms}$ ramped cross-polarisation from ${ }^{1} \mathrm{H}$ to ${ }^{19} \mathrm{~F}$ at a proton nutation frequency of $40 \mathrm{kHz}$ followed by SPINAL-64 proton decoupling ${ }^{11}$ at a field of $83 \mathrm{kHz}$ during signal acquisition. The spectrum of lyophilised sample is the result of averaging 2048 transients and the spectrum of solid FBTA is the result of averaging 128 transients. The protondecoupled ${ }^{19} \mathrm{~F}$ spectrum of the hydrated membrane sample at $30{ }^{\circ} \mathrm{C}$ was obtained with direct excitation of ${ }^{19} \mathrm{~F}$ using a $4.2 \mu \mathrm{s} 90^{\circ}$ pulse and is the result of averaging 64 transients. The proton-coupled spectrum was acquired under the same conditions but omitting proton irradiation, and is the result of averaging 2048 transients.

The ${ }^{1} \mathrm{H}-{ }^{19} \mathrm{~F}$ polarisation-inversion spin-exchange at the magicangle (PISEMA) spectra were obtained using the basic pulse sequence described by $\mathrm{Wu}$ et $a l^{12}$ For the lyophilised sample, an initial $2.5 \mathrm{~ms} 90^{\circ}$ pulse on ${ }^{1} \mathrm{H}$ was followed by $2 \mathrm{~ms}$ ramped crosspolarisation from ${ }^{1} \mathrm{H}$ to ${ }^{19} \mathrm{~F}$ at a proton nutation frequency of $40 \mathrm{kHz}$. Spin exchange at the magic-angle was achieved using frequency switched Lee-Goldberg cross-polarisation at a proton field of $62.5 \mathrm{kHz}$ and matched ${ }^{19} \mathrm{~F}$ field of $76 \mathrm{kHz}$. The spectrum was the result of $32 t_{1}$ increments in the indirect dimension, with averaging of 512 transients per increment. For the hydrated sample at $30{ }^{\circ} \mathrm{C},{ }^{19} \mathrm{~F}$ was excited directly using a $4.2 \mu \mathrm{s} 90^{\circ}$ pulse and 16 transients were averaged for each of $128 t_{1}$ increments.

\section{Computational details}

Optimisation of the molecular geometry and calculation of the NMR parameters was performed using the CASTEP density functional theory code ${ }^{13}$ employing the GIPAW algorithm, ${ }^{14}$ which allows the reconstruction of the all-electron wave function in the presence of a magnetic field. The CASTEP calculations employed the generalised gradient approximation (GGA) PBE functional ${ }^{15}$ and core-valence interactions were described by ultrasoft pseudopotentials. ${ }^{16}$ In the geometry optimisations for the single molecule and for the crystal structure, all atomic positions were allowed to vary. The optimisation of the methanol solvate crystal structure was carried out using the G06 semiempirical dispersion correction scheme, ${ }^{17}$ with the unit cell parameters allowed to vary. Both the geometry optimisation and NMR calculation for this structure were carried out with a $k$-point spacing of $0.052 \pi \AA^{-1}$ and a planewave energy cut-off of 50 Ry. For the isolated molecule, the geometry optimisation and NMR calculation were performed also using a planewave energy cut-off of $50 \mathrm{Ry}$ $(680 \mathrm{eV})$, but calculations were performed using a planewave energy cut-off of $50 \mathrm{Ry}(680 \mathrm{eV})$ and due to the large cell size, a single $k$-point at the fractional coordinate $(0.25,0.25,0.25)$ in reciprocal space for integration over the Brillouin zone. The calculations generate the absolute shielding tensor $(\sigma)$ and diagonalisation of the symmetric part of $\sigma$ yields as eigenvalues the principal components $\sigma_{X X}, \sigma_{Y Y}$ and $\sigma_{Z Z}$ and their orientations in the molecular frame are given by the eigenvectors.

The Euler angles defining the orientation of the individual ${ }^{1} \mathrm{H}-{ }^{19} \mathrm{~F}$ dipolar vectors in the chemical shift principal axis 
system were calculated using a $\mathrm{C}$ program written specifically for the purpose. Briefly, the angles were calculated from the atomic coordinates of the optimised FBTA molecule expressed in a systematically varied CSA axis system with the origin at fluorine. Simulations of protein-coupled and PISEMA spectra were performed using SIMPSON. ${ }^{18}$

\section{Results and discussion}

\section{Theoretical considerations}

Considering a fluorinated molecule in a lipid bilayer, the large ${ }^{19} \mathrm{~F}$ chemical shift anisotropy (CSA) is the most convenient measureable NMR property that carries information on the motional properties and orientation of the molecule. In the absence of motional averaging, the CSA tensor $\delta$ has three principal components, $\delta_{11}, \delta_{22}$ and $\delta_{33}$, where $\left(\delta_{11}-\delta_{\text {iso }}\right) \geq\left(\delta_{33}-\delta_{\text {iso }}\right) \geq\left(\delta_{22}-\delta_{\text {iso }}\right)$ and $\delta_{\text {iso }}$ is the isotropic chemical shift derived according to $\delta_{\text {iso }}=\left(\delta_{11}+\delta_{22}+\delta_{33}\right) / 3$. The principal values are directed along three orthogonal principal axes. For a static solid, the magnitude of the anisotropy is defined as $\Delta \delta_{\text {stat }}=\delta_{11}-\delta_{\text {iso }}$. For a molecule in a lipid bilayer, rapid anisotropic reorientation at a frequency greater than the static ${ }^{19} \mathrm{~F}$ CSA (typically $>30 \mathrm{kHz}$ ) scales the anisotropy in a predictable way. Rapid molecular reorientation includes lateral diffusion within the plane of the lipid bilayer and/ or vesicular tumbling when the vesicle radius $r$ is sufficiently small, together represented by a single rotational correlation time: ${ }^{19}$

$$
\frac{1}{\tau_{\mathrm{c}}}=\frac{6}{r^{2}}\left(D_{t}+D_{\mathrm{diff}}\right)
$$

where $D_{\mathrm{t}}$ is the diffusion constant for tumbling of a spherical vesicle and $D_{\text {diff }}$ is the rate of lateral diffusion. Additionally, rotational diffusion of the molecule involves rapid reorientation about a principal axis collinear with the bilayer normal; the azimuthal angle $\alpha$ and polar angle $\beta$ define the orientation of the molecular rotational axis in the CSA principal axis system (Fig. 1A). Rotational motions about the two axes orthogonal to the bilayer normal are usually slower and restricted in amplitude because of the lateral compression forces that hold the membrane assembly together. These additional excursions are represented by an order parameter, $S_{\text {mol }}$, which takes values from 0-1.0 $\left(S_{\mathrm{mol}}=0\right.$ represents isotropic rotation and $S_{\mathrm{mol}}=1.0$ indicates rotation about the principal axis only). An additional contribution to motional averaging can arise internally within FBTA by rotation about the bond between the fluoroaromatic and aniline ring systems. The energy barrier to free rotation is predicted to be high and in the region of $160 \mathrm{~kJ} \mathrm{~mol}^{-1}$, according to molecular mechanics calculations, and so internal rotations were not initially considered in the data analysis. It will be shown later that the NMR data are not consistent with internal rotation.

When lateral diffusion, vesicle tumbling and internal motions are not significant, the observed averaged ${ }^{19} \mathrm{~F}$ CSA, $\Delta \delta_{\mathrm{av}}$, is given by $^{20,21}$

$$
\Delta \delta_{\mathrm{av}}=0.5 S_{\mathrm{mol}} \Delta \delta_{\text {stat }}\left(3 \cos ^{2} \beta-1-\eta \sin ^{2} \beta \cos 2 \alpha\right)
$$

where the asymmetry parameter $\eta=\left(\delta_{22}-\delta_{33}\right) / \Delta \delta_{\text {stat }}$. Fig. 1B illustrates an example of the relationship between $\Delta \delta_{\text {obs }}, \alpha$ and $\beta$ for a molecule rotating in a lipid bilayer, for arbitrary values of $\delta_{11}, \delta_{22}$ and $\delta_{33}$ and $S_{\mathrm{mol}}=1.0$ to represent rotation about the principal axis only.

Fig. 1 highlights two points to consider when using scaled CSA values to determine the orientation of the axis of molecular rotation within lipid bilayers. First, it is necessary to know the orientation of the chemical shift principal axis system in the molecular reference frame. Good predictions are now possible
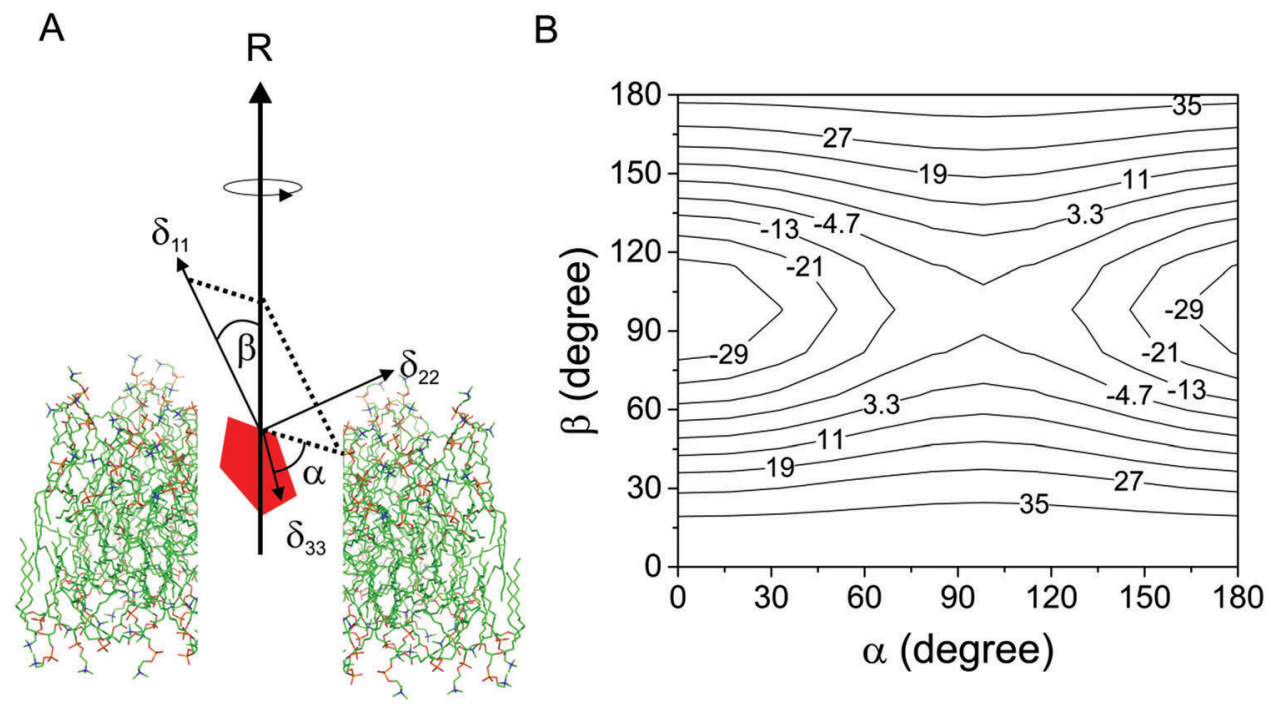

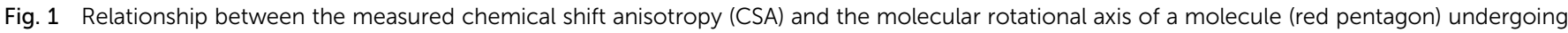

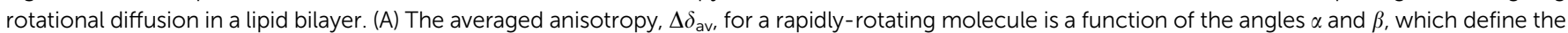

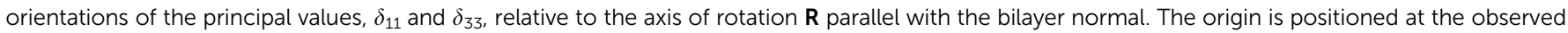

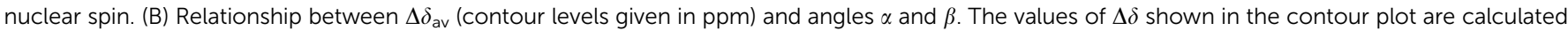

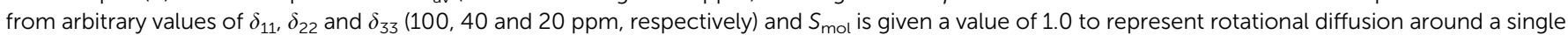
axis with no additional motional fluctuations. 
using quantum chemistry calculations, such as density functional theory (DFT), which have been used as the basis for interpreting the NMR spectra of molecules within lipid bilayers. ${ }^{6,8}$ Close agreement between the calculated and the experimentally-measured principal values is usually taken as an indicator of the reliability of the predicted orientation of the tensor elements in the molecular frame. However, as such calculations are performed on isolated molecules or on molecules within a crystal lattice, they may not accurately reflect the bilayer environment. Secondly, it is clear from Fig. 1B that a continuum of $\alpha$ and $\beta$ values can account for the measured value of $\Delta \delta_{\mathrm{av}}$ and so the direction of the rotational axis in the molecular frame cannot be determined unambiguously from CSA information alone. Supplementary NMR measurements, such as ${ }^{19} \mathrm{~F}-{ }^{13} \mathrm{C}$ dipolar couplings or ${ }^{2} \mathrm{H}$ quadrupolar analysis of deuterated sites within the same molecule, have been shown to reduce the ambiguity but require lengthy measurement times and/or isotope enrichment. ${ }^{6}$

\section{CSA analysis of FBTA in lipid bilayers}

FBTA was incorporated into multilamellar vesicles (MLV) of chain-perdeuterated dimyristoylphosphatidylcholine (DMPC-d ${ }_{54}$ ) at a lipid to FBTA molar ratio of 20:1 and then the mixture was lyophilised to enable the static ${ }^{19} \mathrm{~F} \mathrm{CSA}\left(\right.$ i.e., $\left.\Delta \delta_{\text {stat }}\right)$ to be measured in the lipid environment. Fig. 2B shows the proton-decoupled ${ }^{19} \mathrm{~F}$ spectrum of the lyophilised material, from which were extracted values of $\delta_{11}, \delta_{22}$ and $\delta_{33}$ (Table 1). The line shape is consistent with an axially asymmetric CSA tensor expected for FBTA in a static powder environment. The bilayers were then hydrated with phosphate buffer $\left(10 \mathrm{mM}\right.$ in $\left.\mathrm{D}_{2} \mathrm{O}\right)$ and a second spectrum was recorded at $30{ }^{\circ} \mathrm{C}\left(\sim 7{ }^{\circ} \mathrm{C}\right.$ above the chain melting temperature of DMPC) (Fig. 2C). The ${ }^{19} \mathrm{~F}$ line shape now indicates a scaled and axially symmetric CSA brought about by rapid (i.e., $>50 \mathrm{kHz}$, the static powder line width) motional averaging of the CSA; the value of $\Delta \delta_{\text {av }}$ lies between $1.05 \mathrm{ppm}$ and $1.25 \mathrm{ppm}$ according to comparisons with simulated spectra (Fig. 2D). Lateral diffusion and vesicle tumbling are not expected to influence the line shape because of the large size and low curvature of MLVs, ${ }^{19,23}$ but if FBTA disrupts the lipid bilayer and promotes the formation of smaller vesicles and/or non-bilayer structures then contributions of these modes of motion may be significant. A ${ }^{31} \mathrm{P}$ NMR spectrum of the lipid head groups in the same sample gives an indication of the influence of lateral diffusion. The full width of the measured ${ }^{31} \mathrm{P}$ powder pattern, $\Delta \delta=\left(\delta_{\|}-\delta_{\perp}\right)$, is close to $45 \mathrm{ppm}$ (Fig. S1, ESI $\dagger$ ), which is the value expected for large MLVs in which averaging of the static ${ }^{31} \mathrm{P}$ CSA of the choline head groups is principally by rotational motion. ${ }^{19,23}$ Simulated spectra indicate that additional rapid lateral diffusion (or vesicle tumbling) resulting in a mean angular displacement of the lipid from its initial position within the plane of the bilayer would reduce the powder line width, ${ }^{22}$ and a displacement as small as $10^{\circ}$ results in a measureable reduction of $\Delta \delta$. If it is assumed that the rate of lateral diffusion of FBTA is similar to that of DMPC, it is reasonable to neglect the effects of lateral diffusion when analysing the ${ }^{19} \mathrm{~F}$ spectra and focus on the effects of rotational motions.

Combinations of angles $\alpha$ and $\beta$ that are consistent with $1.05 \leq \Delta \delta_{\mathrm{av}} \leq 1.25 \mathrm{ppm}$ were calculated from eqn (2) for values of $S_{\mathrm{mol}}$ of 1.0 and 0.1 . For each $S_{\mathrm{mol}}$ value, two distributions of angle combinations are observed, as expected, each distribution forming a sinusoidal continuum (Fig. 2E). The combinations of permitted angles are clearly sensitive to $S_{\text {mol }}$ and so this variable must be taken into account in the calculations later. A value of 0.80 for $S_{\text {mol }}$ is typical of the order parameters determined for ergosterol and cholesterol in lipid bilayers. ${ }^{6,8}$

\section{DFT calculations}

Reliable calculation or measurement of the shielding tensor orientation in the molecular frame is required to extract the details of the FBTA molecular rotational axis from the NMR data. DFT calculations of the chemical shielding tensor values and orientation in the molecular frame were performed using the crystal structure coordinates for FBTA crystallised as a methanol solvate (orthorhombic, space group Pbca; Fig. S2, ESI $\dagger$ ). The calculations generate the absolute shielding tensor $(\sigma)$ with principal components $\sigma_{X X}>\sigma_{Y Y}>\sigma_{Z Z}$, and the calculated isotropic shielding, $\sigma_{\text {ic }}$, is given by $(1 / 3) \operatorname{Tr} \sigma$. The calculated chemical shift values are obtained by subtracting $\sigma_{X X}, \sigma_{Y Y}$ and $\sigma_{Z Z}$ from a reference shielding value defined here as

$$
\sigma_{\text {ref }}=\delta_{\text {iso }}+1 / 3\left(\sigma_{X X}+\sigma_{Y Y}+\sigma_{Z Z}\right)
$$

where $\delta_{\text {iso }}$ is the experimentally-measured isotropic shift value. The narrow line widths in the ${ }^{19} \mathrm{~F}$ NMR spectrum obtained with magic angle spinning are consistent with a predominantly highly-ordered crystalline material with $Z^{\prime}=1$, but a smaller broader component indicates the presence of another form of FBTA as a minor impurity (Fig. S3, ESI $\dagger$ ). The crystalline component was estimated to represent approximately $95 \%$ of the total FBTA, by integration after subtraction of a broaderlinewidth spectrum of FBTA recrystallized from ethanol:water (Fig. S3B and C, ESI $\dagger$ ). Table 1 compares the calculated ${ }^{19}$ F NMR shift values with the values measured from the static powder spectrum of the methanol solvate after correction for the minor FBTA contaminant (Fig. S3, panel D, ESI $\dagger$ ). There is some discrepancy between the measured and calculated anisotropy and asymmetry parameter. The crystal lattice is unrepresentative of the lipid bilayer environment, however, and neither the calculated nor experimental CSA values for the crystalline form agree with the values measured for FBTA in DMPC- $\mathrm{d}_{54}$ (Table 1). DFT calculations could not be performed on FBTA in a lipid bilayer because the atomistic details are unknown, so instead a new set of NMR parameters were calculated for an isolated FBTA molecule centred in a cubic cell of dimensions $20 \times 20 \times 20 \AA$ to ensure no interactions between periodic replicas. The calculations on both the crystalline and isolated molecules indicated that $\delta_{22}$ is directed along the $\mathrm{C}-\mathrm{F}$ bond and $\delta_{33}$ is parallel with the normal of the aromatic ring (Fig. 3A). The calculated principal components of $\delta$ (Table 1) are somewhat different from the measured values and may reflect the inability to replicate the bilayer environment in the calculations.

\section{Experimental validation of DFT calculations}

The discrepancy between the calculated and measured chemical shift components reduces confidence in the reliability of the 

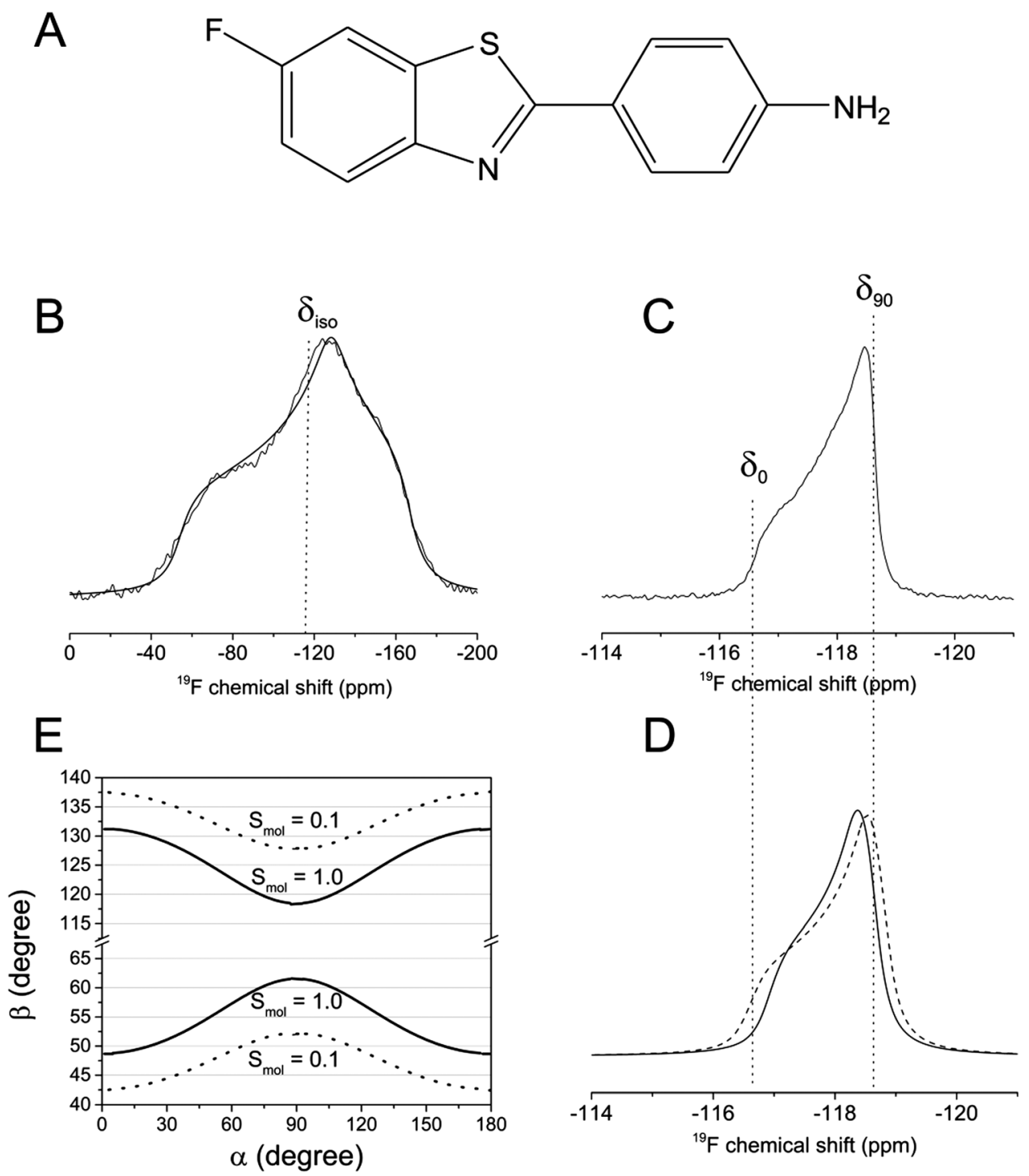

Fig. 2 Static, proton-decoupled ${ }^{19} \mathrm{~F}$ NMR spectra of FBTA in DMPC- $d_{54}$ bilayers. (A) Chemical structure of FBTA. (B) Powder spectrum of FBTA in lyophilised bilayers, with the calculated isotropic chemical shift, $\delta_{\text {iso, }}$ indicated. (C) Dynamically averaged powder pattern in hydrated bilayers at $30{ }^{\circ} \mathrm{C}$, where $\delta_{0}$ and $\delta_{90}$ are the ${ }^{19} \mathrm{~F}$ chemical shifts for bilayer orientations at $0^{\circ}$ and $90^{\circ}$ to the magnetic field. (D) Simulated axially symmetric powder patterns for $\Delta \delta_{\mathrm{av}}$ values of $1.05 \mathrm{ppm}$ (solid line) and $1.25 \mathrm{ppm}$ (dashed line), representing the lower and upper limits of the best fits to the experimental spectrum. (E) Permitted combinations of angles, $[\alpha, \beta]$, consistent with values of $\Delta \delta=1.15 \pm 0.10 \mathrm{ppm}$, when $S_{\mathrm{mol}}=1.0$ (solid lines) and $S_{\mathrm{mol}}=0.1$ (dashed lines).

Table 1 Experimental and calculated ${ }^{19} \mathrm{~F}$ static chemical shift tensor elements for FBTA. Calculated principal values were obtained from the computed shielding tensor elements and are defined as $\delta_{11}=\sigma_{\text {ref }}-\sigma_{Z Z,} \delta_{22}=\sigma_{\text {ref }}-\sigma_{Y Y}$ and $\delta_{33}=\sigma_{\text {ref }}-\sigma_{X X}$

\begin{tabular}{llllll}
\hline Method (environment) & $\delta_{11}(\mathrm{ppm})$ & $\delta_{22}(\mathrm{ppm})$ & $\delta_{33}(\mathrm{ppm})$ & $\delta_{\text {iso }}(\mathrm{ppm})$ & $\Delta \delta_{\text {stat }}(\mathrm{ppm})$ \\
\hline Experimental (methanol solvate) $^{a}$ & -64.3 & -121.8 & -158.7 & -114.9 & 50.6 \\
Calculated (methanol solvate) $^{b}$ & -41.9 & -117.9 & -185.0 & -114.9 & 73.0 \\
Experimental (lyoph. bilayer) $^{c}$ & -57.3 & -134.8 & -162.5 & -118.2 & 0.73 \\
Calculated (single molecule) $^{c}$ & -36.4 & -133.9 & -183.7 & -118.2 & 0.92 \\
\end{tabular}

${ }^{a}$ Recrystallised from methanol. ${ }^{b}$ Calculated with $\sigma_{\text {ref }}=140.3 \mathrm{ppm}$. Computed values of $\sigma_{\text {ref }}, \sigma_{X X}, \sigma_{Y Y}$ and $\sigma_{Z Z}$ are averages for the 8 molecules in the crystal lattice. ${ }^{c}$ Calculate with $\sigma_{\text {ref }}=145.9 \mathrm{ppm}$.

calculated chemical shift tensor geometry that is needed in the analysis. To test the validity of the calculated tensor orientation we employed a ${ }^{1} \mathrm{H}^{-19} \mathrm{~F}$ PISEMA NMR experiment, ${ }^{12}$ which correlates the chemical shift with the ${ }^{1} \mathrm{H}^{19}{ }^{19} \mathrm{~F}$ dipolar coupling constant for each molecular orientation in a powder distribution. In the lyophilised membrane sample, FBTA is distributed randomly with individual molecules separated by the lipid host. Each ${ }^{19} \mathrm{~F}$ spin of FBTA is dipolar coupled predominantly to three neighbouring proton spins within the same aromatic ring, with coupling constants $d_{\mathrm{HF}}$ of $6433 \mathrm{~Hz}, 6433 \mathrm{~Hz}$ and $1241 \mathrm{~Hz}$ 

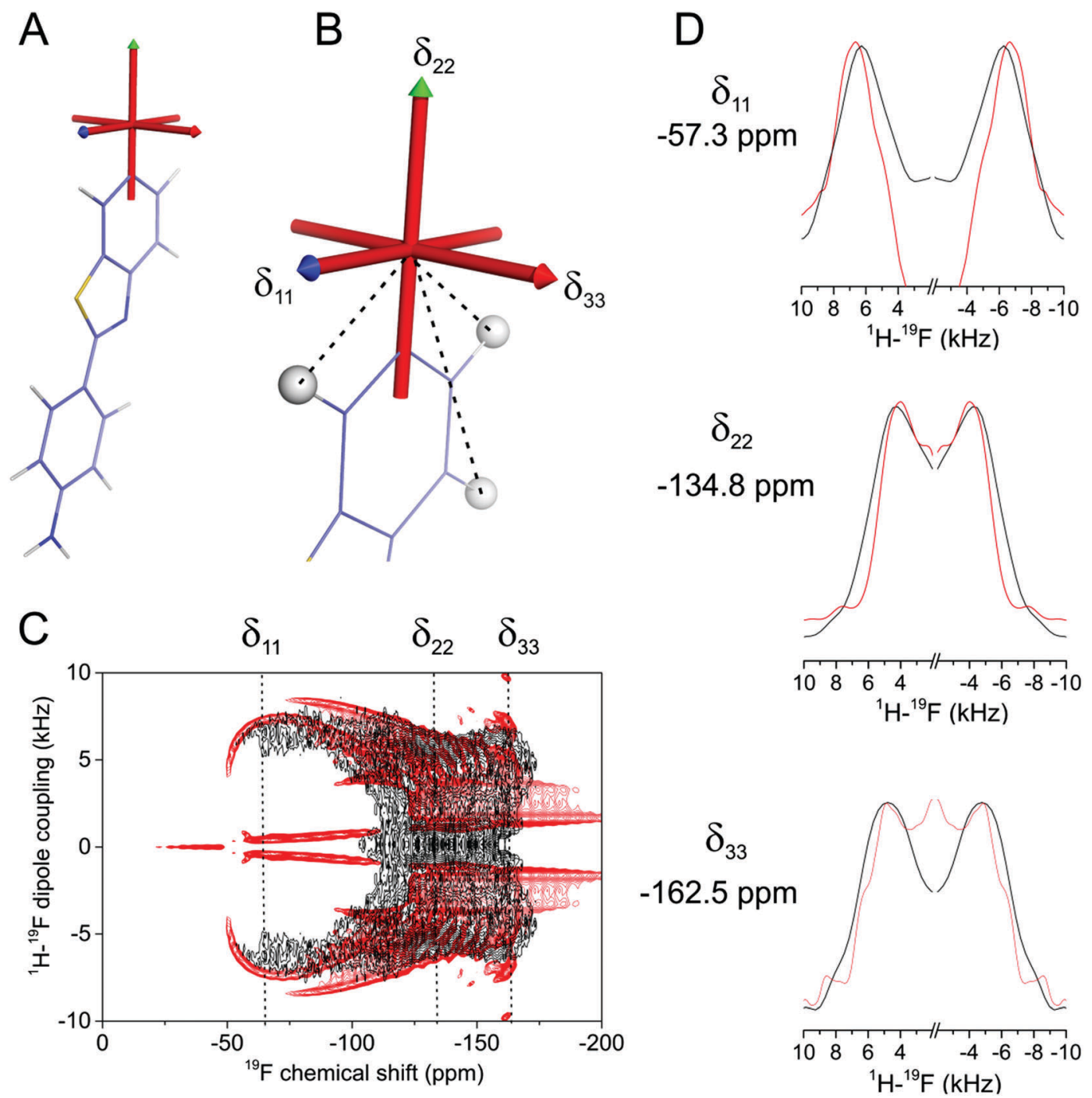

Fig. 3 Orientation of the chemical shift principal axis system of FBTA in the molecular frame. (A) Computed shielding tensor directions in the molecular frame, calculated for an isolated molecule using CASTEP. (B) Expansion of (A) showing $\delta_{22}$ parallel with the $\mathrm{F}-\mathrm{C}$ bond and $\delta_{33}$ normal to the plane of the aromatic ring. Dashed lines indicate the ${ }^{1} \mathrm{H}^{19}{ }^{19} \mathrm{~F}$ dipolar vectors in the $\delta_{11}-\delta_{22}$ plane, at angles (and $\mathrm{H}-\mathrm{F}$ distances) of $34^{\circ}(2.6 \AA), 119^{\circ}(4.4 \AA)$ and $146^{\circ}(2.6 \AA)$. (C) Experimental ${ }^{1} \mathrm{H}-{ }^{19} \mathrm{~F}$ PISEMA NMR spectrum under static conditions (black) of FBTA in lyophilised DMPC-d $\mathrm{d}_{54}$ bilayers overlaid with a simulated spectrum (red) calculated from the $\mathrm{H}-\mathrm{F}$ distances and the orientation of the principal axes shown in (B). (D) Vertical slices through the experimental and calculated spectra at chemical shifts corresponding to (top to bottom) $\delta_{11}, \delta_{22}$ and $\delta_{22}$.

determined from the fixed internuclear distances in the optimised structure (Fig. 3B). The use of chain-perdeuterated DMPC prevents couplings between lipid acyl chain protons and ${ }^{19} \mathrm{~F}$ of FBTA that may otherwise complicate the analysis. In a static powder sample, the dipolar coupling for each molecular orientation is given by $d_{\mathrm{HF}} \times\left(3 \cos ^{2} \theta-1\right) / 2$, where $\theta$ is the angle between the dipolar vector and the magnetic field. The corresponding ${ }^{19} \mathrm{~F}$ chemical shift for each molecular orientation is determined by the orientation of the ${ }^{19} \mathrm{~F}$ CSA tensor in a laboratory frame in which the $z$-axis is parallel with the magnetic field. The shape of the PISEMA spectrum is thus sensitive to the relative orientations of the CSA tensor and the ${ }^{1} \mathrm{H}^{-}{ }^{19} \mathrm{~F}$ dipolar vectors in the same molecular frame, and the spectrum can be readily predicted for the structurally-invariant spin system of FBTA. Fig. 3C shows an experimental ${ }^{1} \mathrm{H}-{ }^{19} \mathrm{~F}$ PISEMA spectrum of FBTA in lyophilised
DMPC- $\mathrm{d}_{54}$ superimposed with a simulated spectrum based on the values of $d_{\mathrm{HF}}$ and the calculated orientation of the CSA tensor in the molecular frame (i.e., $\delta_{22}$ directed along the $\mathrm{C}-\mathrm{F}$ bond and $\delta_{33}$ normal to the aromatic ring plane. Visual inspection reveals a good agreement between experimental and calculated spectra (Fig. 3C and D), but, to test the sensitivity of the spectrum to deviations of the tensor orientation, further simulations were performed in which the each of the principal axes were varied by $\pm 20^{\circ}$ from the initial predicted positions. Even these small deviations resulted in a visibly poorer agreement with the experimental spectrum (Fig. S4, ESI $\dagger$ ). Simulated spectra in which $\delta_{11}$ is directed along the $\mathrm{C}-\mathrm{F}$ bond or normal to the aromatic ring deviate significantly from the observed spectrum (Fig. S5, ESI $\dagger$ ) The PISEMA spectrum is thus consistent with the calculated tensor orientations. 


\section{Orientational information from ${ }^{1} \mathrm{H}-{ }^{19} \mathrm{~F}$ dipolar couplings}

Next we attempted to reduce the ambiguity in the $[\alpha, \beta]$ angles determined from the CSA analysis. As dipolar couplings are also scaled predictably by molecular rotation, it was reasoned that line shape analysis of proton-coupled ${ }^{19} \mathrm{~F}$ spectra would reduce the ambiguity inherent in the CSA measurement alone. Simulations of proton-coupled 1D spectra for several allowed values of $\alpha$ and $\beta$ (i.e., which produce the continuous lines for $\Delta \delta_{\mathrm{av}}=1.15 \pm 0.10 \mathrm{ppm}$ in Fig. $2 \mathrm{E}$ ) show that the ${ }^{19} \mathrm{~F}$ line shape is sensitive to the different combinations of angles (Fig. 4, left hand spectra). In fact, because of molecular symmetry and the dominance of dipolar couplings of the protons ortho to ${ }^{19} \mathrm{~F}$, the combinations of angles $[\alpha, \beta],[180-\alpha, \beta],[\alpha, 180-\beta]$ and $[180-\alpha, 180-\beta]$ all result in similar line shapes. Simulations of rotationally-averaged ${ }^{1} \mathrm{H}-{ }^{19} \mathrm{~F}$ PISEMA spectra also reveal sensitivity to the same $[\alpha, \beta]$ combinations (Fig. 4 , right hand spectra).

Further experimental ${ }^{19} \mathrm{~F}$ NMR spectra of FBTA in DMPC- $\mathrm{d}_{54}$ bilayers were obtained in the presence of ${ }^{1} \mathrm{H}^{-19} \mathrm{~F}$ dipolar couplings.

\section{A ${ }^{1} \mathrm{H}$-coupled ${ }^{19} \mathrm{~F} \quad$ B ${ }^{1} \mathrm{H}-{ }^{19} \mathrm{~F}$ PISEMA}
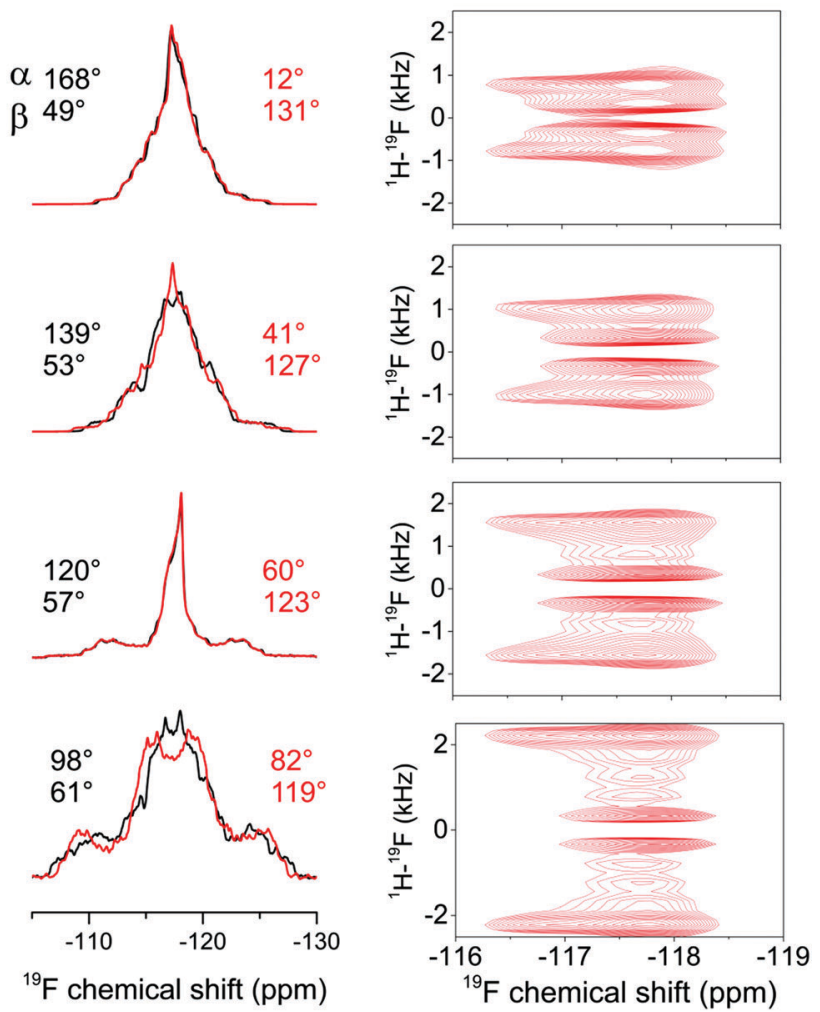

Fig. 4 Analysis of the rotational axis orientation of FBTA in hydrated, liquid-crystalline DMPC- $d_{54}$ bilayers. (A) Simulated static, proton-coupled ${ }^{19} \mathrm{~F}$ spectra for a four-spin $\left({ }^{19} \mathrm{~F}\right.$ and $\left.3 \times{ }^{1} \mathrm{H}\right)$ system undergoing rapid rotation as described in Fig. 1A. Simulations are based on the calculated CSA principal value orientations in the molecular frame from Fig. 3B with three rotationally averaged ${ }^{1} \mathrm{H}-{ }^{19} \mathrm{~F}$ dipolar couplings projected onto the principal rotational axis, with a scaling factor $S_{\text {mol }}$ of 0.80 representing rotational excursions about the axes orthogonal to the bilayer normal. Simulated spectra are shown for 8 combinations of $8 \times \alpha$ and $8 \times \beta$ values, each combination being consistent with the observed $\Delta \delta_{\text {av }}$ range in Fig. $2 \mathrm{E}$. (B) Simulated ${ }^{1} \mathrm{H}-{ }^{19} \mathrm{~F}$ PISEMA spectra for the same $[\alpha, \beta]$ angles shown in red in $\mathrm{A}$.
Table 2 The upper and lower limits of $S_{\mathrm{mol}}, \alpha$ and $\beta$ values that are consistent with $\Delta \delta_{\mathrm{av}}=1.15 \pm 0.10$ and the $1 \mathrm{D}$ proton-coupled ${ }^{19} \mathrm{~F} \mathrm{NMR}$ spectrum and ${ }^{1} \mathrm{H}-{ }^{19} \mathrm{~F}$ PISEMA spectrum in Fig. 5 . Note that the values of $\alpha$ and $\beta$ represent the upper and lower boundaries along a continuum, and not all possible angle combinations between the upper and lower limits are permitted

\begin{tabular}{llll}
\hline$S_{\text {mol }}$ & {$[\alpha, \beta]_{\text {lower }}$} & {$[\alpha, \beta]_{\text {mid }}$} & {$[\alpha, \beta]_{\text {upper }}$} \\
\hline 1.0 (upper) & {$\left[153^{\circ}, 49.5^{\circ}\right]$} & {$\left[0^{\circ}, 47.3^{\circ}\right]$} & {$\left[27^{\circ}, 49.5^{\circ}\right]$} \\
& {$\left[153^{\circ}, 130.5^{\circ}\right]$} & {$\left[0^{\circ}, 132.7^{\circ}\right]$} & {$\left[27^{\circ}, 130.5^{\circ}\right]$} \\
0.8 (best) & {$\left[168^{\circ}, 49.6^{\circ}\right]$} & {$\left[0,48.5^{\circ}\right]$} & {$\left[12^{\circ}, 49.6^{\circ}\right]$} \\
& {$\left[168^{\circ}, 130.4^{\circ}\right]$} & {$\left[0,131.5^{\circ}\right]$} & {$\left[12^{\circ}, 130.4^{\circ}\right]$} \\
0.7 (lower) & {$\left[147^{\circ}, 50.7^{\circ}\right]$} & {$\left[ \pm 8^{\circ}, 48.3^{\circ}\right]$} & {$\left[33^{\circ}, 50.7^{\circ}\right]$} \\
& {$\left[147^{\circ}, 129.7^{\circ}\right]$} & {$\left[ \pm 8^{\circ}, 131.7^{\circ}\right]$} & {$\left[33^{\circ}, 129.7^{\circ}\right]$}
\end{tabular}

The 1D proton-coupled spectrum was compared with a series of simulated spectra to find the combinations of $S_{\mathrm{mol}}, \alpha$ and $\beta$ giving the best agreement. $S_{\text {mol }}$ was varied from 0-1.0 in increments of 0.1 and applied as a scaling factor for the three ${ }^{1} \mathrm{H}-{ }^{19} \mathrm{~F}$ dipolar couplings $d_{\mathrm{HF}}$ and used to calculate the values of $\alpha$ and $\beta$ that are consistent with the measured $\Delta \delta_{\mathrm{av}}$ of $1.15 \pm 0.10 \mathrm{ppm}$. The variance between the simulated and experimental spectra was calculated for each set of $S_{\mathrm{mol}}, \alpha$ and $\beta$ values (Fig. S6 and S7, $\mathrm{ESI} \dagger)$. Simulations for $S_{\mathrm{mol}}$ values of less than 0.7 did not produce good agreement with the experimental spectrum and were discounted (Fig. S7, ESI $\dagger$ ). The remaining acceptable values of the three variables are given in Table 2 . The best overall agreement corresponds to $S_{\mathrm{mol}}=0.8$ and angle combinations of $\left[\alpha=0 \pm 12^{\circ}\right.$, $\left.\beta=49^{\circ} \pm 0.5^{\circ}\right]$ and $\left[\alpha=0 \pm 12^{\circ}, \beta=130.5^{\circ} \pm 0.5^{\circ}\right]$ (Fig. 5A). Good agreement is also seen between an experimental ${ }^{1} \mathrm{H}-{ }^{19} \mathrm{~F}$ PISEMA spectrum of the same sample and a simulated spectrum calculated using the parameter set $\left[S_{\mathrm{mol}}=0.8, \alpha=12^{\circ}, \beta=131^{\circ}\right]$ (Fig. 5B). Together, the combination of ${ }^{19} \mathrm{~F}$ CSA and ${ }^{1} \mathrm{H}-{ }^{19} \mathrm{~F}$ coupling measurements has restrained the rotational diffusion axis of FBTA to within a narrow range of orientations in the molecular frame.

Finally we calculated how internal rotation of the fluoroaromatic ring would influence the spectra. Rapid internal rotation about an axis parallel with the bond linking the two ring systems scales the ${ }^{19} \mathrm{~F}$ CSA to $\sim 40 \mathrm{kHz}$ in the absence of other motion, with the principal axis of the axially symmetric tensor directed along the axis of internal rotation. Further averaging will occur as a result of molecular rotational diffusion about the axis collinear with the bilayer normal, and the observed scaling of the CSA to $\Delta \delta_{\mathrm{av}}=1.15 \pm$ $0.1 \mathrm{ppm}$ depends on a single angle, $\theta$, between the internal rotation axis and the molecular rotational axis (Fig. S8, ESI $\dagger$ ). For an initial condition of $S_{\mathrm{mol}}=1.0$ it is found that angle $\theta=51^{\circ}$ or $129^{\circ}$, which by coincidence is similar to the angle $\beta$ calculated in the absence of internal rotation. However, the corresponding rotationally scaled values of $d_{\mathrm{HF}}$ are all less than $150 \mathrm{~Hz}$, which are lower than the values $(979 \mathrm{~Hz}, 798 \mathrm{~Hz}$ and $188 \mathrm{~Hz}$ ) required for a good fit to the 1D and PISEMA spectra. For $S_{\mathrm{mol}}<1.0$, the scaled values of $d_{\mathrm{HF}}$ are even lower. It is unlikely, therefore, that rapid internal motions contribute to the observed spectra.

\section{Conclusions}

In summary, we present a new NMR approach, combining ${ }^{19} \mathrm{~F}$ CSA and ${ }^{1} \mathrm{H}-{ }^{19} \mathrm{~F}$ dipolar coupling measurements, for determining 

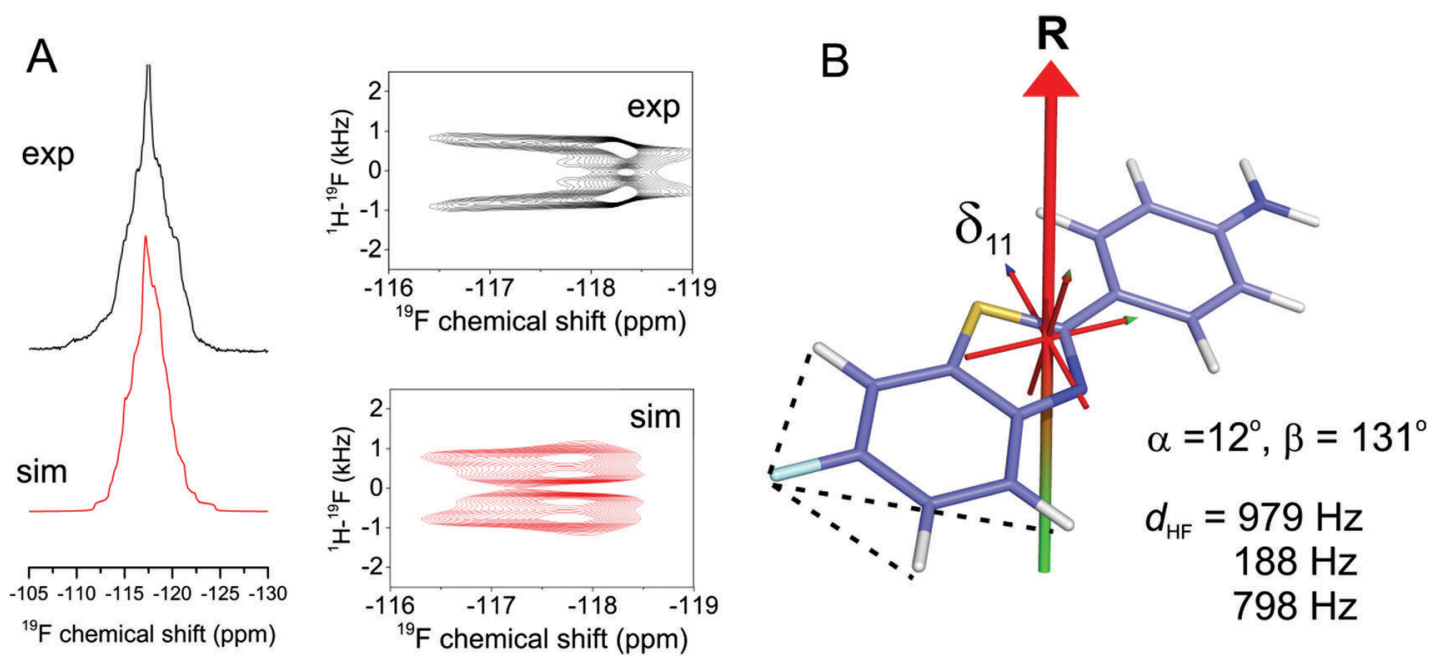

Fig. 5 Additional restraints on the axis of rotation of FBTA in hydrated DMPC- $d_{54}$ bilayers at $30{ }^{\circ} \mathrm{C}$. (A) Experimental proton-coupled $1 \mathrm{D}{ }^{19} \mathrm{~F}$ and ${ }^{1} \mathrm{H}-{ }^{19} \mathrm{~F}$ PISEMA NMR spectra (black) and simulated spectrum in closest agreement (red). The simulated spectra correspond to the values of angles $\alpha$ and $\beta$, and coupling constants $d_{\mathrm{HF}}$ shown at the bottom right of the figure. (B) Representation of FBTA rotating about a single axis inclined at the angles $\alpha$ and $\beta$ shown. The axis system is shown with the origin coincident with the centre of mass of FBTA. The dotted lines signify the ${ }^{1} \mathrm{H}-{ }^{19} \mathrm{~F}$ couplings. The molecule is predicted to be positioned in a lipid bilayer with the $-\mathrm{NH}_{2}$ group facing toward the lipid-water interface rather than toward the hydrophobic interior.

average molecular orientations within lipid bilayers. This approach can provide detailed information about the biological activity of fluorinated pharmaceutical or diagnostic molecules with minimal chemical preparation. The approach requires NMR probeheads and appropriate frequency filters enabling observation of ${ }^{19} \mathrm{~F}$ whilst simultaneously decoupling protons, but such equipment is now routinely commercially available. In the given example, uncertainties in the average orientation were reduced by the lack of internal rotational of the FBTA molecule and by the narrow range of the order parameter $S_{\text {mol }}$, which restricts angular fluctuations from the principal molecular rotational axis. In the case of more flexible molecules, for which these additional motions may be more significant, further independent NMR constraints such as ${ }^{1} \mathrm{H}^{-13} \mathrm{C}$ dipolar couplings (at natural ${ }^{13} \mathrm{C}$ abundance) may be required to determine the average orientation with more certainty.

We envisage a range of applications beyond the model compound study reported here. It is anticipated that this approach will be useful when comparing the membrane properties of families of drug molecules such as statins, several of which contain fluorine. ${ }^{24}$ Indeed, this class of molecules has been evaluated for use with lipid-nanoparticle based drug-delivery systems (recombinant high-density lipoprotein) ${ }^{25}$ and our approach can report on their properties in these confined environments compared to more expansive lipid bilayers. Changes in drug orientations in the presence of integral membrane proteins such as efflux protein can also be studied using this method.

\section{Abbreviations}

PISEMA

Polarisation inversion spin exchange at the magic angle

CSA REDOR
GIPAW

CASTEP

DMPC

\section{Conflicts of interest}

There are no conflicts to declare.

\section{Acknowledgements}

The work was supported by the British Heart Foundation project grant PG/16/97/32567.

\section{References}

1 B. C. Wang, L. J. Wang, B. Jiang, S. Y. Wang, N. Wu, X. Q. Li and D. Y. Shi, Mini-Rev. Med. Chem., 2017, 17, 683-692.

2 J. Ma, L. Domicevica, J. R. Schnell and P. C. Biggin, Phys. Chem. Chem. Phys., 2015, 17, 19766-19776.

3 Y. Zhou, J. Wang, Z. N. Gu, S. N. Wang, W. Zhu, J. L. Acena, V. A. Soloshonok, K. Izawa and H. Liu, Chem. Rev., 2016, 116, 422-518.

4 J. Barry, M. Fritz, J. R. Brender, P. E. S. Smith, D. K. Lee and A. Ramamoorthy, J. Am. Chem. Soc., 2009, 131, 4490-4498.

5 M. P. Boland and D. A. Middleton, Phys. Chem. Chem. Phys., 2008, 10, 178-185.

6 N. Matsumori, Y. Kasai, T. Oishi, M. Murata and K. Nomura, J. Am. Chem. Soc., 2008, 130, 4757-4766.

7 E. Okamura and M. Nakahara, J. Phys. Chem. B, 1999, 103, 3505-3509.

8 O. Soubias, F. Jolibois, S. Massou, A. Milon and V. Reat, Biophys. J., 2005, 89, 1120-1131. 
9 W. E. Klunk, H. Engler, A. Nordberg, Y. M. Wang, G. Blomqvist, D. P. Holt, M. Bergstrom, I. Savitcheva, G. F. Huang, S. Estrada, B. Ausen, M. L. Debnath, J. Barletta, J. C. Price, J. Sandell, B. J. Lopresti, A. Wall, P. Koivisto, G. Antoni, C. A. Mathis and B. Langstrom, Ann. Neurol., 2004, 55, 306-319.

10 G. M. Sheldrick, Acta Crystallogr., Sect. A: Found. Crystallogr., 2008, 64, 112-122.

11 B. M. Fung, A. K. Khitrin and K. Ermolaev, J. Magn. Reson., 2000, 142, 97-101.

12 C. H. Wu, A. Ramamoorthy and S. J. Opella, J. Magn. Reson., Ser. A, 1994, 109, 270-272.

13 M. D. Segall, P. J. D. Lindan, M. J. Probert, C. J. Pickard, P. J. Hasnip, S. J. Clark and M. C. Payne, J. Phys.: Condens. Matter, 2002, 14, 2717-2744.

14 C. J. Pickard and F. Mauri, Phys. Rev. B: Condens. Matter Mater. Phys., 2001, 63, 245101.

15 J. P. Perdew, K. Burke and M. Ernzerhof, Phys. Rev. Lett., 1996, 77, 3865-3868.

16 J. R. Yates, C. J. Pickard and F. Mauri, Phys. Rev. B: Condens. Matter Mater. Phys., 2007, 76, 024401.
17 S. Grimme, J. Comput. Chem., 2006, 27, 1787-1799.

18 M. Bak, J. T. Rasmussen and N. C. Nielsen, J. Magn. Reson., 2000, 147, 296-330.

19 E. E. Burnell, P. R. Cullis and B. Dekruijff, Biochim. Biophys. Acta, 1980, 603, 63-69.

20 J. H. Davis and M. Auger, Prog. Nucl. Magn. Reson. Spectrosc., 1999, 35, 1-84.

21 B. Bechinger, Biochim. Biophys. Acta, Biomembr., 1999, 1462, 157-183.

22 R. F. Campbell, E. Meirovitch and J. H. Freed, J. Phys. Chem., 1979, 83, 525-533.

23 P. M. Macdonald, Q. Saleem, A. Lai and H. H. Morales, Chem. Phys. Lipids, 2013, 166, 31-44.

24 M. Schachter, Fundam. Clin. Pharmacol., 2005, 19, 117-125.

25 R. Duivenvoorden, J. Tang, D. P. Cormode, A. J. Mieszawska, D. Izquierdo-Garcia, C. Ozcan, M. J. Otten, N. Zaidi, M. E. Lobatto, S. M. van Rijs, B. Priem, E. L. Kuan, C. Martel, B. Hewing, H. Sager, M. Nahrendorf, G. J. Randolph, E. S. G. Stroes, V. Fuster, E. A. Fisher, Z. A. Fayad and W. J. M. Mulder, Nat. Commun., 2014, 5, 11. 